\title{
Central DNA Flap Sequence
}

National Cancer Institute

\section{Source}

National Cancer Institute. Central DNA Flap Sequence. NCI Thesaurus. Code C13724.

In Lentivirus, a central strand displacement event consecutive to central initiation and termination of plus strand synthesis creates a plus strand overlap: the central DNA flap. This feature promotes efficient nuclear import and viral replication in non-dividing cells. (from Cell 2000;101:173-85) 